tivaria. O componente emergente, relacionado justamente ao processo de trabalho desses profissionais é essencial. Diríamos que o processo de reestruturação discutido na obra em pauta, depende da articulação de uma escolha estratégica amplamente difundida pelo corpo profissional. Nesse contexto, enfoques de gestão hospitalar regional como o da démarche statégique 4,5,6 seriam extremamente pertinentes, por respeitarem determinadas configurações das organizações profissionais, por objetivarem envolver os profissionais de saúde, especialmente os médicos, no gerenciamento estratégico da coordenação hospitalar e considerarem o ambiente externo (parcerias).

É imprescindível salientar a importância da obra para os leitores brasileiros, sejam estes ligados aos serviços ou à academia, e recomendar sua tradução para o português.

\section{Elizabeth Artmann}

Francisco Javier Uribe Rivera

Escola Nacional de Saúde Pública Sergio Arouca,

Fundação Oswaldo Cruz, Rio de Janeiro, Brasil.

artmann@ensp.fiocruz.br

uribe@ensp.fiocruz.br

1. Champágne F. La capacite de gérer le changement dans les organisations de santé. http://www.hc-sc. gc.ca/francais/pdf/romanow/39_Champagne_ F.pdf (acessado em 17/Jul/2006).

2. Minzberg H. Structure et dynamique des Organisations. Paris: Les Éditions d'Organisation; 1998.

3. Rivera FJU. Análise estratégica em saúde e gestão pela escuta. Rio de Janeiro: Editora Fiocruz; 2003.

4. Crémadez M, Grateau F. Le management stratégique hospitalier. Paris: InterEditions/Masson; 1997.

5. Artmann E. Demárche stratégique (gestão estratégica hospitalar): um enfoque que busca mudança através da comunicação e da solidariedade em rede [Tese de Doutorado]. Campinas: Departamento de Medicina Preventiva, Faculdade de Ciências Médicas; 2002.

6. Rivera FJU. O enfoque de integração estratégica de Crémadez e Grateau e a gestão de recursos humanos e tecnológicos. Rev Adm Pública 1998; 32 : 179-207.

VACINAS, SOROS \& IMUNIZAÇÕES NO BRASIL. Buss PM, Temporão JG, Carvalheiro JR, organizadores. Rio de Janeiro: Editora Fiocruz; 2005. 420 pp.

ISBN: 85-7541-060-1

Este é um livro interessantíssimo. Trata de assunto fundamental para a Saúde Pública, os imunobiológicos, fornecendo informações detalhadas e pouco conhecidas sobre a história e o estado da arte da pesquisa e desenvolvimento desses produtos no Brasil, com textos bem escritos e tendo como autores pesquisadores e gestores de Ciência \& Tecnologia (C\&T) diretamente envolvidos com essa temática. Deve ser lido e poderá ser de interesse para um público bastante amplo. Dito isso, passemos ao detalhamento da estrutura temática adotada e às principais questões que a leitura dos diferentes capítulos provocaram, já alertando também que a extensão do livro inevitavelmente implicará a omissão de questões importantes, face aos limites colocados para uma (simples) resenha.
A concepção do livro se insere no contexto de um conjunto de iniciativas desenvolvidas nos últimos anos, na área da saúde e da ciência e tecnologia, para o desenvolvimento de políticas capazes de transformar o nosso desempenho na Pesquisa, Desenvolvimento \& Inovação de tecnologias para a saúde. O livro está estruturado em seis partes complementares: (1) A Magnitude do Problema das Doenças Imunopreveníveis no Brasil; (2) Complexo Produtivo: Política e Regulação de Vacinas e Soros no Brasil; (3) Prospecção em Vacinas: Resultados do Projeto Inovação; (4) Pesquisa, Desenvolvimento e Inovação em Vacinas no Brasil; (5) Complexo Produtivo da Saúde no Brasil: A Experiência das Instituições; e (6) Vacinas e Imunizações: A Experiência Internacional.

Na primeira parte, o capítulo inicial, um texto elaborado pela Secretaria de Vigilância à Saúde (SVS) do Ministério da Saúde apresenta uma síntese da situação de prevenção e controle das doenças transmissíveis no Brasil, mostrando que um grupo de doenças tem tendência decrescente nos indicadores epidemiológicos, algumas por serem atualmente imunopreveníveis e as vacinas fazerem parte do Programa Nacional de Imunização (PNI), outras pela implantação de programas de controle e tratamento efetivos e fatores sociais, ambientais e epidemiológicos. No entanto, muitas doenças transmissíveis apresentam ainda perfis epidemiológicos com situações persistentes ou mesmo de avanço, incluindo algumas (meningites, tuberculose, febre amarela, hepatites) para as quais já existem vacinas, mas que ainda não foi alcançada, por diferentes razões, uma imunoprevenção efetiva. O segundo capítulo apresenta síntese de estudo maior realizado por um grupo de pesquisadores de diferentes instituições, de projeção da carga de doença no Brasil, de 1998 a 2013, prevendo uma tendência de declínio da participação das doenças infecciosas e tropicais, nos anos perdidos por incapacidade (DALY) na população brasileira. No entanto, como os próprios autores apontam, essa metodologia de estudo do impacto das doenças sobre as populações ainda está sujeita a muitos questionamentos, e não tem sido muito utilizada ultimamente. Essa primeira parte procura demonstrar que as doenças infecciosas são um problema de saúde atual e importante, ao contrário do que se previa algumas décadas atrás, e que há uma necessidade e potencial de demanda para o maior número possível de vacinas efetivas quantas puderem ser desenvolvidas e incorporadas aos sistemas de saúde.

A segunda parte, que trata do complexo produtivo, das políticas e da regulação das vacinas e soros, está organizada em cinco capítulos. O primeiro deles, escrito por Carlos Augusto Grabois Gadelha, faz uma análise muito instigante da (preocupante) situação atual do complexo industrial da saúde no Brasil, identificando, para os diferentes segmentos (equipamentos e materiais, vacinas, reagentes para diagnósticos e fármacos, medicamentos e hemoderivados), seus fatores críticos de competitividade, fontes de vantagens competitivas no Brasil, nichos tecnológicos e de mercado e políticas para uma (maior) competitividade. Em síntese, o Brasil teria um complexo industrial da saúde frágil e vulnerável, e para poder alterar essa condição "torna-se necessário um novo padrão de intervenção e uma aproximação entre o universo da ciência, da tecnologia e da inovação e o 
universo da política social" (p. 88). O segundo capítulo, de autoria de Edmundo Gallo \& Ivan Coelho, adota também uma perspectiva ampla ao tratar da regulação econômica e financiamento do complexo produtivo da saúde e o papel do Ministério da Saúde, propondo políticas regulatórias mais diretas do Estado (e do próprio Ministério) sobre o complexo industrial da saúde.

Os terceiro, quarto e quinto capítulos da segunda parte focam questões específicas relativas aos imunobiológicos. José Gomes Temporão, Márcia Vieira Leite Nascimento e Maria de Lourdes de Sousa Maia analisam, de forma muito interessante, o PNI, sua evolução histórica, impressionante dimensão atual e desafios (integração, sustentabilidade e expansão). As questões específicas da vigilância sanitária nos imunobiológicos são discutidas por Dario Pinto Miranda \& Claudio Maierovitch P. Henriques, que identificam como um ponto de inflexão para a vigilância sanitária em geral e dos imunobiológicos em particular, os problemas detectados na qualidade dos produtos brasileiros no final dos anos 70 e início da década seguinte, a partir dos quais foi implantado Programa Nacional de Qualidade, que foi de fato importante para que uma nova fase se iniciasse. Um dos desdobramentos importantes foi a criação, em 1981, do Instituto Nacional de Controle de Qualidade em Saúde (INCQS), na Fundação Oswaldo Cruz (FIOCRUZ), e sua participação nos processos necessários para a garantia continuada da qualidade dos imunobiológicos é analisada por André Luis Gemal e Eduardo Chaves Leal, de forma detalhada, apontando a participação do INCQS nas últimas décadas, nas seis funções essenciais a serem desenvolvidas pelas autoridades de vigilância sanitária: avaliação dos requisitos mínimos de qualidade por meio de análise laboratorial, liberação dos lotes, registro, inspeção das BPF, farmacovigilância e autorização para estudos clínicos.

A parte III do livro, considerada pelos organizadores como medular, trata dos resultados do Projeto Inovação na Prospecção em Vacinas, em cinco capítulos. O primeiro, escrito por Julie Milstein, pesquisadora do Center for Vaccine Development, University of Maryland School of Medicine, analisa, utilizando abordagem muito atual, o desenvolvimento tecnológico de vacinas, com projeções para 2015. A autora faz uma síntese do contexto brasileiro, identificando como problemas importantes para o desenvolvimento tecnológico nacional em vacinas a reduzida disponibilidade de recursos humanos e financeiros em C\&T e a natureza exclusivamente pública da indústria e do mercado de vacinas, e aponta algumas vantagens competitivas que poderiam ser exploradas. Em uma perspectiva global, considera que são atualmente decisivas para o desenvolvimento tecnológico em vacinas as questões da eficiência na produção, o acompanhamento dos avanços na biotecnologia, a regulação da qualidade dos produtos, a legislação sobre a propriedade intelectual das inovações e os mecanismos de estabelecimento dos preços dos produtos. O mercado mundial de vacinas está cada vez mais controlado pelas grandes indústrias farmacêuticas, mas apresenta diferenças na sua dinâmica, por comparação aos medicamentos, com uma presença mais importante de agências internacionais na definição e controle da demanda e o estabelecimento de redes de parcerias complexas.
O segundo capítulo da parte III tem como autor Manuel Limonta, Diretor de Biotecnologia do Instituto de Hematologia e Imunologia cubano, e apresenta os resultados da Avaliação Tecnológica da produção de imunobiológicos no Brasil e de definição de nichos de atuação. Foram analisados os laboratórios nacionais responsáveis pela produção de vacinas e soros incluídos no PNI: Bio-Manguinhos/FIOCRUZ no Rio de Janeiro, Instituto Butantan em São Paulo, Fundação Ataulpho de Paiva (FAP) no Rio de Janeiro, Instituto de Tecnologia do Paraná (TECPAR), por meio de entrevistas com gestores e pesquisadores, análises de dados secundários e observação direta, o que permitiu uma caracterização detalhada da sua estrutura física e de recursos humanos, Pesquisa \& Desenvolvimento $(P \& D)$ e produção, em uma perspectiva temporal. Foram elaborados diagnósticos de dimensões estratégicas (perfil tecnológico, adequação das instalações e processos, perfil da mão-de-obra e auto-sustentabilidade tecnológica) e são apresentadas sugestões para o enfrentamento dos problemas identificados.

No terceiro capítulo, José Vitor Bontempo \& Rosiceli Barreto G. Baetas desenvolvem uma análise da potencialidade da $P \& D$ e estratégias de inovação em vacinas no Brasil. A partir do Diretório Lattes de Pesquisadores e Grupos de Pesquisa do Conselho Nacional de Desenvolvimento Científico e Tecnológico (CNPq), foram identificados laboratórios e pesquisadores envolvidos, de alguma forma, com P\&D de vacinas no Brasil, e enviados questionários para a identificação das etapas de desenvolvimento das vacinas em que atuam, fatores críticos de sucesso e dificuldades enfrentadas. Foram também entrevistados os produtores de vacinas e estudadas suas competências para inovação. Os resultados obtidos são muito interessantes e como conclusão destacam-se como pontos positivos a preocupação crescente dos produtores com a capacitação e inovação, e o número importante de grupos com capacitação em pesquisa básica. Como fatores limitantes, são apontados a insuficiência de recursos para $P \& D$ e limitações de infraestrutura para os produtores, e a grande dificuldade dos grupos de pesquisadores ultrapassarem as etapas iniciais de pesquisa básica e pré-desenvolvimento das vacinas. No quarto capítulo, aprofunda-se a análise dos produtores de vacinas no Brasil, e José Cezar Castanhar, Francisco Marcelo Barone \& Paulo Robeto Motta analisam os dados da avaliação gerencial que realizaram com os gestores dos laboratórios, nas dimensões institucionais e propriamente gerenciais, e concluem com recomendações para mudanças estratégicas, principalmente na gestão econômico-financeira.

O último capítulo da parte III, que tem como autores José da Rocha Carvalheiro, José Gomes Temporão, Akira Homma \& Hisako Gondo Higashi, apresenta uma versão do Programa Inovacina que resume as conclusões do amplo processo de discussão entre gestores, produtores e acadêmicos, os stakeholders da P\&D em vacinas no Brasil. Foram elaborados propósitos, objetivos e instrumentos que concretizam os planos de ação para os componentes principais do Programa Nacional de Competitividade em Vacinas - Definição de Políticas e Organização da Produção, Modernização e Certificação do Parque Produtivo, Avaliação e Regulação e Desenvolvimento e Inovação -, e desenvolvidos quadros de prioridades para os próximos 3, 5 e 10 anos. 
Na parte IV, organizada em quatro capítulos, são apresentadas iniciativas recentes de apoio à pesquisa, desenvolvimento e inovação em vacinas pelo CNPq, FIOCRUZ e Instituto Butantan. O capítulo sobre fomento à pesquisa e desenvolvimento em vacinas e soros no CNPq, de autoria de Manoel Barral Netto e Erney Plessmann Camargo, analisa as informações referentes ao período 2001-2002 de recursos gastos no financiamento de projetos e bolsas para estudos de vacinas e soros pelo CNPq, indicando uma demanda reduzida e fragmentada nessas áreas, no período (a partir de 2004 essa situação parece ter se alterado).

A análise do desenvolvimento tecnológico em vacinas e outros insumos para a saúde na FIOCRUZ, desenvolvida por Euzenir Nunes Sarno, Jussara Nascimento, Wim Maurits Sylvain Degrave e Paulo Marchiori Buss, destaca a priorização da $P \& D$ na instituição a partir de 2001, com a implementação do Programa de Desenvolvimento Tecnológico em Insumos em Saúde (PDTIS), do Programa de Desenvolvimento Tecnológico em Saúde Pública (PDTSP) e a continuidade do Programa de Apoio à Pesquisa em Saúde (PAPES), de financiamento projetos de pesquisa com recursos da FIOCRUZ. O PDTIS é financiado com recursos oriundos do resultado financeiro da produção de insumos na FIOCRUZ (em 2003 os gastos totais realizados foram de cerca de 9,5 milhões de reais), tem no desenvolvimento de vacinas um de seus principais objetivos e está estruturado em quatro redes Medicamentos e Bioinseticidas, Vacinas Recombinantes e DNA, Genoma e Proteoma e Diagnóstico - e já apoiou mais de 58 projetos que têm como objetivo principal otimizar as pesquisas caracterizadas como pré-desenvolvimento. Componente essencial para completar o fortalecimento da $\mathrm{P} \& \mathrm{D}$, será a implantação do Centro de Desenvolvimento Tecnológico em Saúde (CDTS), previsto para entrar em operação em 2007, cuja proposta é detalhada por Eduardo Azeredo Costa, Carlos Médicis Morel e Paulo Marchiori Buss, e que deverá fornecer a infra-estrutura necessária para que as pesquisas possam resultar em processos de desenvolvimento tecnológico.

O último capítulo da parte IV, que tem como autores Isaias Raw, Hisako Gondo Hisashi \& Otávio Azevedo Mercadante, apresenta um panorama detalhado da pesquisa e desenvolvimento em vacinas no Instituto Butantan. Destaca a importância estratégica da criação do Centro de Biotecnologia, onde têm sido priorizados aqueles projetos de interesse mais imediato para a saúde humana e as demandas do PNI, buscando oferecer, por meio de estratégias diversas e alianças com outros Centros de Pesquisas nacionais e internacionais e indústrias, vacinas eficazes a preços compatíveis.

A parte $\mathrm{V}$ apresenta o complexo produtivo público de vacinas e imunobiológicos no Brasil, com capítulos dedicados ao Instituto Butantan, Bio-Manguinhos/FIOCRUZ, TECPAR e Centro de Produção e Pesquisa de Imunobiológicos (CPPI). Os textos, elaborados por diretores dessas instituições, apresentam trajetórias institucionais específicas, mas têm em comum a identificação de significativos desenvolvimentos nos últimos vinte anos na produção nacional de vacinas e imunobiológicos, mas concordam com as análises apresentadas em capítulos anteriores sobre o complexo produtivo, ao identificar dificuldades estruturais para a sustentabilidade do seu desenvol- vimento e concorrência com as empresas privadas multinacionais. Na parte $\mathrm{V}$, bastante reduzida, estão esboçadas algumas experiências internacionais recentes que procuram atenuar os efeitos da lógica de mercado sobre o desenvolvimento e distribuição das vacinas e seus efeitos perversos sobre a saúde das populações pobres.

Em conclusão, e retomando a afirmativa inicial, esse livro apresenta informações preciosas e múltiplos pontos de vista, que permitem conhecer de forma aprofundada o estado da arte das vacinas, soros e imunizações no Brasil.

Hillegonda Maria Dutilh Novaes

Faculdade de Medicina,

Universidade de São Paulo, São Paulo, Brasil.

hidutilh@usp.br

BIOÉTICA: RISCOS E PROTEÇÃO. Schramm FR, Rego S, Braz M, Palácios $M$, organizadores. Rio de Janeiro: Editora UFRJ/Editora Fiocruz; 2005. 256 pp.

ISBN: 85-7108-293-6

O livro Bioética: Riscos e Proteção, lançado em 2005 pela Editora UFRJ e pela Editora Fiocruz, é uma excelente obra organizada competentemente por Fermin Roland Schramm, Sergio Rego, Marlene Braz e Marisa Palácios, é leitura obrigatória por explorar as interfaces entre a bioética de proteção, os desafios da biotecnociência, questionamentos e propostas que envolvem a saúde pública.

Fruto de Seminário que congregou especialistas das mais variadas disciplinas, tendo como tema central a ética, aplicada às novas relações e questões geradas na construção do conceito de Bioética de Proteção.

A bioética surgiu como uma ponte entre a filosofia e as ciências biológicas. Essa ponte tornou-se necessária para fazer frente às conseqüências do desenvolvimento tecnológico sobre o meio ambiente e a sobrevivência da espécie humana. Hoje há necessidade de destacar seu interesse nos aspectos ligados à saúde pública por analisar, refletir e prescrever determinada conduta moral partindo de um diálogo pluralista.

A bioética de proteção considera que o governo tem o compromisso de proteger todos os membros da sociedade, diante de qualquer intervenção que não seja estável, independente da estrutura de Estado, a consciência da vulnerabilidade enquanto condição humana, e que a filosofia e a política propostas às sociedades devem garantir a proteção de seus cidadãos contra a violência, pobreza e quaisquer tipos de violação aos direitos humanos 1 .

Sua preocupação particular é com os riscos e a vulnerabilidade, que as pessoas vulneráveis correm de serem prejudicadas pelas conseqüências das ações realizadas por profissionais da saúde 2 .

As políticas públicas de distribuição de recursos em saúde, com as restrições de acesso a determinadas tecnologias, dão origem a dilemas éticos relacionados aos princípios de beneficência, não maleficência, autonomia, justiça e dignidade da natureza humana para tratá-lo como fim e não como meio, na tomada de decisão diante desses dilemas quando devem ser considerados valores sociais, éticos e critérios técnicos. 